\title{
The projections of the zeros of exponential polynomials with complex frequencies
}

\author{
A. Mas \\ Departamento de Matemáticas \\ Universidad Autónoma de Madrid \\ 28049 Madrid, Spain \\ E-mail: alejandro.mas@uam.es \\ J.M. Sepulcre \\ Departamento de Matemáticas \\ Universidad de Alicante \\ 03080 Alicante, Spain \\ E-mail: JM.Sepulcre@ua.es
}

\begin{abstract}
In this paper we study the global distribution of zeros of exponential polynomials with complex coefficients and frequencies. For any $P(z)$ in some class of such polynomials, we show that the closure of the projection of $P^{-1}(0)$ on a certain line is a finite union of disjoint segments. We describe this set, in particular we discuss the case where it consists of one segment.
\end{abstract}

\section{Introduction}

For each integer $n \geq 2$, let

$$
P(z)=m_{1} e^{\alpha_{1} z}+m_{2} e^{\alpha_{2} z}+\ldots+m_{n} e^{\alpha_{n} z}, z \in \mathbb{C},
$$

be an exponential polynomial with non-zero complex coefficients $m_{1}, m_{2}, \ldots$, $m_{n}$ and distinct complex frequencies $\alpha_{1}, \alpha_{2}, \ldots, \alpha_{n}$. It is known that $P(z)$

2010 Mathematics Subject Classification: Primary 30C15, 30D20; Secondary 30Axx.

Key words and phrases: Zeros of entire functions, Exponential polynomials, Zeros of exponential polynomials, Entire functions. 
is an entire function having infinitely many zeros located in certain specific regions of the complex plane. The distribution of the zeros of such a polynomial has been extensively studied during the twentieth century, mostly in the first third of that century, due to its relation with some general problems of the expansion theory for linear differential equations. See, for example, [6], [9], [10], [15], [16] and [17].

If $Q(z)=m_{1} e^{\alpha_{1} z}+m_{2} e^{\alpha_{2} z}+\ldots+m_{n} e^{\alpha_{n} z}$ is an exponential polynomial of type (1.1) with real frequencies $\alpha_{1}<\alpha_{2}<\ldots<\alpha_{n}$, it is not difficult to prove that the numbers

$$
a_{Q}:=\inf \{\operatorname{Re} z: Q(z)=0\} \quad \text { and } \quad b_{Q}:=\sup \{\operatorname{Re} z: Q(z)=0\}
$$

are both finite, and so the zeros of $Q(z)$ lie in the vertical strip bounded by the lines $a_{Q}+i \mathbb{R}$ and $b_{Q}+i \mathbb{R}$. These bounds allow us to define an interval $I_{Q}:=\left[a_{Q}, b_{Q}\right]$, called the critical interval, which contains the closure of the set of the real projections of the zeros of $Q(z)$, denoted by

$$
R_{Q}:=\overline{\{\operatorname{Re} z: Q(z)=0\}} .
$$

To our best knowledge, the first work on the existence of zeros of $Q(z)$ (with real frequencies) arbitrarily close to any line contained in certain substrips of its critical strip was made by Moreno in 1973 [8, Main theorem]. It is also necessary to cite Avellar and Hale [1, Theorem 3.1], who introduced in 1980 a criterion to decide whether a real number is in the set $R_{Q}$. The study of density properties of the real projections of the zeros of exponential polynomials with real frequencies has recently become a topic of increasing interest (see for example $[2,3,4,5,7,11,12,13,14]$ ).

In this paper, we first analyse in Section 2 the global position of the zeros of the general class of exponential polynomials of type (1.1). The rest of the paper is devoted to generalize the procedure used in [7] in order to extract similar results in a more general case (specially in the case where exponential polynomials satisfy conditions presented in Notation 3.4). In short, for this type of polynomials, our paper provides a description of the set $R_{P}$ defined as the closure of the projection of $P^{-1}(0)$ on a certain line (see Proposition 3.2 and Theorem 3.3) and, in fact, its main results can be summarized by:

i) The set $R_{P}$ is the union of a finite amount of disjoint nondegenerate line segments (see Theorem 3.3);

ii) If the coefficients of $P(z)$ have the same absolute value, then the set $R_{P}$ consists of one segment (see Corollary 3.5). 


\section{The global position of the zeros of expo- nential polynomials}

The distribution of the set of zeros of exponential polynomials with polynomial coefficients and complex frequencies was studied by several authors such as Pólya [9, p. 286] or Langer [6, Theorem 8]. If $P(z)=m_{1} e^{\alpha_{1} z}+$ $m_{2} e^{\alpha_{2} z}+\ldots+m_{n} e^{\alpha_{n} z}$ is an exponential polynomial, let $Z_{P}$ denote the set of its zeros, and let $C_{P}$ denote the convex hull $\operatorname{conv}\left\{\overline{\alpha_{1}}, \ldots, \overline{\alpha_{n}}\right\}$. Then the zeros of $P(z)$ lie in half-strips in the directions of the exterior normals to $C_{P}$. For completeness we will provide the proof of this result. First, we consider some particular cases.

Let

$$
Q(z)=m_{1} e^{\alpha_{1} z}+m_{2} e^{\alpha_{2} z}+\ldots+m_{n} e^{\alpha_{n} z}, z \in \mathbb{C},
$$

where $n \geq 2$, be an exponential polynomial with non-zero complex coefficients $m_{1}, m_{2}, \ldots, m_{n}$ and distinct real frequencies $\alpha_{1}<\alpha_{2}<\ldots<\alpha_{n}$. Thus $Q(z)$ has the same set of zeros as

$$
Q_{1}(z)=\frac{Q(z)}{m_{1} e^{\alpha_{1} z}}=1+\frac{m_{2}}{m_{1}} e^{\left(\alpha_{2}-\alpha_{1}\right) z}+\ldots+\frac{m_{n}}{m_{1}} e^{\left(\alpha_{n}-\alpha_{1}\right) z},
$$

which is an exponential polynomial with increasing positive frequencies $0<$ $\alpha_{2}-\alpha_{1}<\ldots<\alpha_{n}-\alpha_{1}$. Since

$$
\lim _{x \rightarrow-\infty} Q_{1}(x+i y)=1, \quad \lim _{x \rightarrow+\infty} \frac{Q_{1}(x+i y)}{\frac{m_{n}}{m_{1}} e^{\left(\alpha_{n}-\alpha_{1}\right)(x+i y)}}=1,
$$

for any value of $y$, there exist $x_{1}<0<x_{2}$ such that

$$
\left|Q_{1}(z)-1\right|<1, \text { for all } z \text { with } \operatorname{Re} z \leq x_{1},
$$

and

$$
\left|\frac{Q_{1}(z)}{\frac{m_{n}}{m_{1}} e^{\left(\alpha_{n}-\alpha_{1}\right)(x+i y)}}-1\right|<1 \text {, for all } z \text { with } \operatorname{Re} z \geq x_{2} .
$$

Hence $Q_{1}(z)$ has no zero neither in the half-plane $\operatorname{Re} z \leq x_{1}$ nor in the halfplane $\operatorname{Re} z \geq x_{2}$. Consequently all the zeros of $Q(z)$, which are the same as zeros of $Q_{1}(z)$, are situated in the vertical strip $\left\{z \in \mathbb{C}: x_{1}<\operatorname{Re} z<x_{2}\right\}$.

Let

$$
R(z)=m_{1} e^{\beta_{1} z}+m_{2} e^{\beta_{2} z}+\ldots+m_{n} e^{\beta_{n} z}, n \geq 2,
$$

be an exponential polynomial with non-zero complex coefficients $m_{1}, m_{2}, \ldots$, $m_{n}$ and with distinct complex frequencies $\beta_{1}, \beta_{2}, \ldots, \beta_{n}$ whose principal arguments are equal. Thus it can be written in the form

$$
R(z)=m_{1} e^{\alpha_{1} e^{i \phi} z}+m_{2} e^{\alpha_{2} e^{i \phi} z}+\ldots+m_{n} e^{\alpha_{n} e^{i \phi} z}
$$


for some $\phi \in \mathbb{R}$ and $0 \leq \alpha_{1}<\alpha_{2}<\ldots<\alpha_{n}$. Then the exponential polynomial $Q(z)=R\left(e^{-i \phi} z\right)=m_{1} e^{\alpha_{1} z}+\ldots+m_{n} e^{\alpha_{n} z}$ is of type (2.1) and therefore there exist $x_{1}<0<x_{2}$ such that

$$
Z_{Q} \subset\left\{z \in \mathbb{C}: x_{1}<\operatorname{Re} z<x_{2}\right\}
$$

More so, it is clear that $Z_{R}=e^{-i \phi} Z_{Q}$. In fact, if $z_{0} \in Z_{R}$ then

$$
z_{0} e^{i \phi}=\operatorname{Re} z_{0} \cdot \cos \phi-\operatorname{Im} z_{0} \cdot \sin \phi+i\left(\operatorname{Im} z_{0} \cdot \cos \phi+\operatorname{Re} z_{0} \cdot \sin \phi\right) \in Z_{Q} .
$$

From (2.2) we have that $x_{1}<\operatorname{Re}\left(z_{0} e^{i \phi}\right)<x_{2}$. Hence all the zeros of $R(z)$ are in the strip

$\left\{z \in \mathbb{C}: x_{1}<\operatorname{Re} z \cdot \cos \phi-\operatorname{Im} z \cdot \sin \phi<x_{2}\right\}=\left\{z \in \mathbb{C}: x_{1}<\operatorname{Re}\left(z e^{i \phi}\right)<x_{2}\right\}$

More generally, let

$$
S(z)=m_{1} e^{\gamma_{1} z}+m_{2} e^{\gamma_{2} z}+\ldots+m_{n} e^{\gamma_{n} z}, n \geq 2,
$$

be an exponential polynomial with non-zero complex coefficients $m_{1}, m_{2}, \ldots$, $m_{n}$ and with distinct aligned (or co-linear) frequencies $\gamma_{1}, \gamma_{2}, \ldots, \gamma_{n}$. Without loss of generality we may assume that there exists $\varphi \in[0, \pi)$ such that the frequencies of $S(z)$ can be written in the form

$$
\gamma_{j}=\gamma_{1}+\alpha_{j} e^{i \varphi}, j=1,2, \ldots, n
$$

with $0=\alpha_{1}<\alpha_{2}<\ldots<\alpha_{n}$. Hence

$$
S(z)=m_{1} e^{\gamma_{1} z}\left(1+\frac{m_{2}}{m_{1}} e^{\alpha_{2} e^{i \varphi} z}+\ldots+\frac{m_{n}}{m_{1}} e^{\alpha_{n} e^{i \varphi} z}\right) .
$$

Notice also that the exponential polynomial $Q(z)=S\left(z e^{-i \varphi}\right)$ is so that $Z_{S}=e^{-i \varphi} Z_{Q}$. Moreover, since the set of zeros of $Q(z)$ coincides with that of the exponential polynomial $1+\frac{m_{2}}{m_{1}} e^{\alpha_{2} z}+\ldots+\frac{m_{n}}{m_{1}} e^{\alpha_{n} z}$, which is of type (2.1), there exist $x_{1}<0<x_{2}$ such that $Z_{Q} \subset\left\{z \in \mathbb{C}: x_{1}<\operatorname{Re} z<x_{2}\right\}$. Consequently, if $z_{0} \in Z_{S}$ then $z_{0} e^{i \varphi} \in Z_{Q}$ and $x_{1}<\operatorname{Re}\left(z_{0} e^{i \varphi}\right)<x_{2}$. That means that all the zeros of $S(z)$ are in the strip

$\left\{z \in \mathbb{C}: x_{1}<\operatorname{Re}\left(z e^{i \varphi}\right)<x_{2}\right\}=\left\{z \in \mathbb{C}: x_{1}<\operatorname{Re} z \cdot \cos \varphi-\operatorname{Im} z \cdot \sin \varphi<x_{2}\right\}$.

We next deal with the general case where we will divide the plane into a finite number of sectors and, subsequently, we will apply the methods above to each of them. 
Before going to this, let us recall the following elementary remark that will be taken into account: if $V=(a, b)$ and $U=(\cos \gamma, \sin \gamma)$ are two vectors in the plane, with $a, b, \gamma \in \mathbb{R}$, so that $0 \leq \theta \leq \frac{\pi}{2}$ is the angle between them, then the length of the orthogonal projection of $V$ on the direction of $U$ is $\|V\| \cos \theta$, and thus the scalar product of $U$ and $V$ coincides with it.

Theorem 2.1. Let

$$
P(z)=m_{1} e^{\alpha_{1} z}+\ldots+m_{n} e^{\alpha_{n} z}, n \geq 2,
$$

be an exponential polynomial of type (1.1) and let $C_{P}=\operatorname{conv}\left\{\overline{\alpha_{1}}, \ldots, \overline{\alpha_{n}}\right\}$. Then all the zeros of $P(z)$ lie in half-strips in the directions of the exterior normals to $C_{P}$.

Proof. Let $l_{1}, l_{2}, \ldots, l_{q}$ be the sides of $C_{P}$ taken in counterclockwise succession with any one as the initial side and, for each $r=1,2, \ldots, q$, let $\overline{\alpha_{r, 1}}, \overline{\alpha_{r, 2}}, \ldots, \overline{\alpha_{r, h_{r}}}$ be the complex conjugates of the frequencies of $P(z)$ which lie on the side $l_{r}$, the succession being again in the counterclockwise sense around the polygon $C_{P}$. First notice that it is possible that some complex conjugates of the frequencies, say $\overline{\alpha_{0,1}}, \overline{\alpha_{0,2}}, \ldots, \overline{\alpha_{0, h_{0}}}$, lie within the polygon $C_{P}$. Finally, we denote as $m_{r, i}$ the coefficient of $P(z)$ associated with $\alpha_{r, i}$. Given $r \in\{1,2, \ldots, q\}$, let $\gamma$ be a real number such that $w_{r-1}+\varepsilon \leq \gamma<w_{r}+\varepsilon$ where $w_{r}$ is the inclination angle of the outer normal to the side $l_{r}$ and $\varepsilon>0$ is sufficiently small. (Considering all the possible values of $r$, note that the set of these sectors completely fills out the complex plane). If $z=|z| e^{i \gamma}$ and $\alpha_{s}=a_{s}+i b_{s} \in \mathbb{C}$, it is worth noting that

$\operatorname{Re}\left(\alpha_{s} e^{i \gamma}\right)=\operatorname{Re}\left(\left(a_{s}+i b_{s}\right)(\cos \gamma+i \sin \gamma)\right)=a_{s} \cos \gamma-b_{s} \sin \gamma=\operatorname{Re}\left(\overline{\alpha_{s}} e^{-i \gamma}\right)$

and

$$
\left|e^{\alpha_{s} z}\right|=\left|e^{\left(a_{s}+i b_{s}\right)|z| e^{i \gamma}}\right|=e^{|z|\left(a_{s} \cos \gamma-b_{s} \sin \gamma\right)}=e^{|z| \operatorname{Re}\left(\overline{\alpha_{s}} e^{-i \gamma}\right)} .
$$

Moreover, $a_{s} \cos \gamma-b_{s} \sin \gamma$ can be expressed as the scalar product of the vectors $\overline{\alpha_{s}}=\left(a_{s},-b_{s}\right)$ and $e^{i \gamma}=(\cos \gamma, \sin \gamma)$. So, by the remark before the theorem, if the term $\operatorname{Re}\left(\overline{\alpha_{s}} e^{-i \gamma}\right)$ is positive, it is represented geometrically by the absolute value of the projection of $\left(a_{s},-b_{s}\right)$ on the direction of $e^{i \gamma}$. Now, from this geometrical interpretation, and since $w_{r-1}+\varepsilon \leq \gamma<w_{r}+\varepsilon$, we have

$$
\operatorname{Re}\left(\overline{\alpha_{s, k}} e^{-i \gamma}\right)<\operatorname{Re}\left(\overline{\alpha_{r, 1}} e^{-i \gamma}\right) \text {, with } s \neq r \text { and } k \in\left\{1,2, \ldots, h_{s}\right\}
$$


Note that if $\operatorname{Re}\left(\overline{\alpha_{s, k}} e^{-i \gamma}\right)<0$ for some $s \neq r$ and $k \in\left\{1,2, \ldots, h_{s}\right\}$, inequality (2.6) is trivially true. Also, if $\gamma=w_{r}$ it is clear that $\operatorname{Re}\left(\overline{\alpha_{r, 1}} e^{-i \gamma}\right)=$ $\operatorname{Re}\left(\overline{\alpha_{r, 2}} e^{-i \gamma}\right)=\ldots=\operatorname{Re}\left(\overline{\alpha_{r, h_{r}}} e^{-i \gamma}\right)$. Now take $z=|z| e^{i \gamma} \in \mathbb{C}$, then

$P(z)=m_{1} e^{\alpha_{1} z}+m_{2} e^{\alpha_{2} z}+\ldots+m_{n} e^{\alpha_{n} z}=\sum_{k=1}^{h_{r}} m_{r, k} e^{\alpha_{r, k} z}+\sum_{s=0, s \neq r}^{q} \sum_{k=1}^{h_{s}} m_{s, k} e^{\alpha_{s, k} z}$

and, by dividing by $m_{r, 1} e^{\alpha_{r, 1} z}$, the set of zeros of $P(z)$ is the same as the set of solutions of the equation

$$
1+\sum_{k=2}^{h_{r}} \frac{m_{r, k}}{m_{r, 1}} e^{\left(\alpha_{r, k}-\alpha_{r, 1}\right) z}+\sum_{s=0, s \neq r}^{q} \sum_{k=1}^{h_{s}} \frac{m_{s, k}}{m_{r, 1}} e^{\left(\alpha_{s, k}-\alpha_{r, 1}\right) z}=0
$$

Let $s \in\{0,1, \ldots, q\}$ with $s \neq r$ and $k \in\left\{1,2, \ldots, h_{s}\right\}$. From (2.5), we have

$$
\left|e^{\left(\alpha_{s, k}-\alpha_{r, 1}\right) z}\right|=e^{|z|\left(\operatorname{Re}\left(\overline{\alpha_{s, k}} e^{-i \gamma}\right)-\operatorname{Re}\left(\overline{\alpha_{r, 1}} e^{-i \gamma}\right)\right)} .
$$

Therefore, we deduce from (2.6) and (2.8) that

$$
\lim _{|z| \rightarrow \infty} e^{|z|\left(\operatorname{Re}\left(\overline{\alpha_{s, k}} e^{-i \gamma}\right)-\operatorname{Re}\left(\overline{\alpha_{r, 1}} e^{-i \gamma}\right)\right)}=0 .
$$

Consequently, noticing (2.7), all but finitely many of the zeros of $P(z)$ are determined by the exponential polynomial $1+\sum_{k=2}^{h_{r}} \frac{m_{r, k}}{m_{r, 1}} e^{\left(\alpha_{r, k}-\alpha_{r, 1}\right) z}$, whose zeros are the same as those of $S_{r}(z)=\sum_{k=1}^{h_{r}} m_{r, k} e^{\alpha_{r, k} z}$. Finally, since the frequencies of $S_{r}(z)$ are aligned (their conjugates lie on the side $l_{r}$ ), we deduce from (2.4) that the zeros of $S_{r}(z)$ are in a half-strip that is parallel to the normal to the side $l_{r}$, i.e. the one which points in the same direction as $w_{r}$. Consequently, by repeating this process for each $r=1,2, \ldots, q$, we obtain the result.

Example 2.2. Consider the exponential polynomial

$$
\begin{aligned}
P(z)=(2+i) e^{2 z}+(-1+ & i) e^{(1.38-1.9 i) z}+3 e^{(3-3.08 i) z}+ \\
& +3 i e^{(4.62-1.9 i) z}+(1+2 i) e^{4 z}+e^{3 z}+i e^{(3-i) z}
\end{aligned}
$$

whose frequencies are $\alpha_{1}=2, \alpha_{2}=1.38-1.9 i, \quad \alpha_{3}=3-3.08 i, \quad \alpha_{4}=$ $4.62-1.9 i, \alpha_{5}=4, \alpha_{6}=3, \alpha_{7}=3-i$. Figure 1 presents the convex hull $C_{P}$ of the set $\left\{\overline{\alpha_{1}}, \overline{\alpha_{2}}, \overline{\alpha_{3}}, \overline{\alpha_{4}}, \overline{\alpha_{5}}, \overline{\alpha_{6}}, \overline{\alpha_{7}}\right\}$, as well as the distribution of those zeros of $P(z)$ whose real and imaginary part is between -30 and 30 . As one can observe, it follows the description given in Theorem 2.1. 

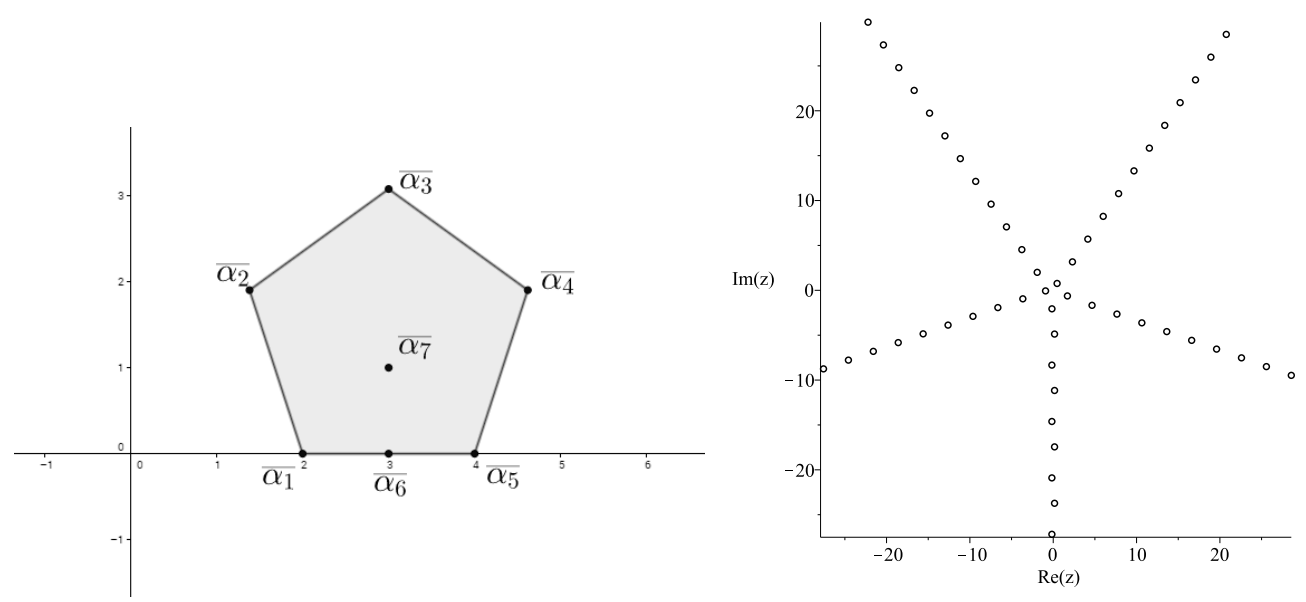

Figure 1: The convex hull $C_{P}$ and the zeros of $P(z)$, respectively, in Example 2.2

\section{Projections of the zeros of exponential poly- nomials}

By Theorem 2.1, given an exponential polynomial $P(z)$ of type (1.1), the study of the properties of its zeros must be carried out in each one of the half-strips where the zeros of $P(z)$ are located. So, let

$$
S(z)=m_{1} e^{\gamma_{1} z}+m_{2} e^{\gamma_{2} z}+\ldots+m_{n} e^{\gamma_{n} z}, n \geq 2,
$$

be an exponential polynomial of type (2.3) with distinct co-linear frequencies $\gamma_{1}, \gamma_{2}, \ldots, \gamma_{n}$. Hence, without loss of generality, there exists $\varphi \in[0, \pi)$ such that $S(z)$ can be written as

$$
m_{1} e^{\left(\gamma_{1}+\alpha_{1} e^{i \varphi}\right) z}+m_{2} e^{\left(\gamma_{1}+\alpha_{2} e^{i \varphi}\right) z}+\ldots+m_{n} e^{\left(\gamma_{1}+\alpha_{n} e^{i \varphi}\right) z}, n \geq 2,
$$

for some $0=\alpha_{1}<\alpha_{2}<\ldots<\alpha_{n}$.

In this section we are interested in the set

$$
R_{S}=\overline{\left\{e^{-i \varphi} \operatorname{Re}\left(z e^{i \varphi}\right): S(z)=0\right\}},
$$

which is the closure of the set of the projections of the zeros of $S(z)$ on the straight line $L_{-\varphi}$ which goes through the origin and has $e^{-i \varphi}$ as director vector. Geometrically, given a straight line $L_{-\varphi+\frac{\pi}{2}}^{x_{0}}$ which has $e^{-i\left(\varphi-\frac{\pi}{2}\right)}$ as director vector and that goes through the point $x_{0} e^{-i \varphi}$, with $x_{0} \in \mathbb{R}$, the point $x_{0} e^{-i \varphi}$ belongs to the set $R_{S}$ when $S(z)$ possesses zeros arbitrarily close to the straight line $L_{-\varphi+\frac{\pi}{2}}^{x_{0}}$. That means that for fixed $\varepsilon>0$ there exists a complex number $z^{*}$ such that $S\left(z^{*} e^{-i \varphi}\right)=0$ and $\left|\sigma^{*}-x_{0}\right|<\varepsilon$, where $\sigma^{*} e^{-i \varphi}$ is the orthogonal projection of $z^{*} e^{-i \varphi}$ on the straight line $L_{-\varphi}$. 
It is worth to notice that an interval of the form $\left(x_{1} e^{-i \varphi}, x_{2} e^{-i \varphi}\right)=\{z \in$ $\left.\mathbb{C}: z=x e^{-i \varphi}, x_{1}<x<x_{2}\right\}$, with $x_{1}<x_{2}$, is contained in $R_{S}$ when every point $x e^{-i \varphi}$ with $x_{1}<x<x_{2}$ is an accumulation point of the set defined by the orthogonal projections of the zeros of $S(z)$ on the straight line $L_{-\varphi}$. That is, if $R_{S}$ is a perfect set, it is immediate that a point $x_{0} e^{-i \varphi}$ is in $R_{S}$ when $x_{0} e^{-i \varphi}$ is an accumulation point of the set defined by the orthogonal projections of the zeros of $S(z)$ on the straight line $L_{-\varphi}$.

Notice that if $\varphi=0$ then the zeros of $S(z)$ can be seen as those of an exponential polynomial with real frequencies, and the set $R_{S}$ corresponds with the closure of the set of the real projections of the zeros of $S(z)$. Hence the expression given in (3.2) generalizes that of (1.2) for the case of exponential polynomials with co-linear frequencies.

Remark 3.1. Let $S(z)=m_{1} e^{\left(\gamma_{1}+\alpha_{1} e^{i \varphi}\right) z}+m_{2} e^{\left(\gamma_{1}+\alpha_{2} e^{i \varphi}\right) z}+\ldots+m_{n} e^{\left(\gamma_{1}+\alpha_{n} e^{i \varphi}\right) z}$ be an exponential polynomial of type (3.1). It is easy to check that

$$
R_{S}=\overline{\left\{e^{-i \varphi} \operatorname{Re} z: S_{1}(z)=0\right\}},
$$

where $S_{1}(z)=1+\frac{m_{2}}{m_{1}} e^{\alpha_{2} z}+\ldots+\frac{m_{n}}{m_{1}} e^{\alpha_{n} z}$. In fact, $S\left(z_{0}\right)=0$ if and only if $S_{1}\left(z_{0} e^{i \varphi}\right)=0$. In particular, $R_{S}$ has the same topological properties as the set $R_{S_{1}}=\overline{\left\{\operatorname{Re} z: S_{1}(z)=0\right\}}$.

In order to investigate properties of $R_{S}$, we shall consider exponential polynomials

$$
S(z)=m_{1} e^{\gamma_{1} z}+m_{2} e^{\left(\gamma_{1}+\alpha_{2} e^{i \varphi}\right) z}+\ldots+m_{n} e^{\left(\gamma_{1}+\alpha_{n} e^{i \varphi}\right) z}, n \geq 2,
$$

of type (3.1) with $0<\alpha_{2}<\ldots<\alpha_{n}$ linearly independent over the field of the rational numbers. If $S_{1}(z)$ is the exponential polynomial which was defined in Remark 3.1, consider the points

$$
a_{S_{1}}=\inf \left\{\operatorname{Re} z: S_{1}(z)=0\right\} \quad \text { and } \quad b_{S_{1}}=\sup \left\{\operatorname{Re} z: S_{1}(z)=0\right\} \text {, }
$$

which are the extreme points of the set $R_{S_{1}}$ and, by [7, Theorem 3], the unique real solutions of the real equations

$$
1=\sum_{j=2}^{n} \frac{\left|m_{j}\right|}{\left|m_{1}\right|} e^{\alpha_{j} x}, \quad \frac{\left|m_{n}\right|}{\left|m_{1}\right|} e^{\alpha_{n} x}=1+\sum_{j=2}^{n-1} \frac{\left|m_{j}\right|}{\left|m_{1}\right|} e^{\alpha_{j} x},
$$

respectively. Thus, from Remark 3.1, the extreme points of the set $R_{S}$ are $a_{S}=a_{S_{1}} e^{-i \varphi}$ and $b_{S}=b_{S_{1}} e^{-i \varphi}$.

The following proposition provides a pointwise characterization of $R_{S}$ when the co-linear frequencies of $S(z)$ satisfy the condition above. 
Proposition 3.2. Let $S(z)=m_{1} e^{\gamma_{1} z}+m_{2} e^{\left(\gamma_{1}+\alpha_{2} e^{i \varphi}\right) z}+\ldots+m_{n} e^{\left(\gamma_{1}+\alpha_{n} e^{i \varphi}\right) z}$, $n>2$, be an exponential polynomial of type (3.1) with $0<\alpha_{2}<\ldots<\alpha_{n}$ linearly independent over the rationals. Then a complex number of the form $\sigma_{0} e^{-i \varphi}$, where $\sigma_{0} \in \mathbb{R}$, is in $R_{S}$ if and only if $\sigma_{0}$ satisfies

$$
1 \leq \sum_{j=2}^{n}\left|\frac{m_{j}}{m_{1}}\right| e^{\alpha_{j} \sigma_{0}} ; \quad\left|\frac{m_{k}}{m_{1}}\right| e^{\alpha_{k} \sigma_{0}} \leq 1+\sum_{j=2, j \neq k}^{n}\left|\frac{m_{j}}{m_{1}}\right| e^{\alpha_{j} \sigma_{0}}, k=2 \ldots, n .
$$

Proof. The polynomial $S_{1}(z)=1+\frac{m_{2}}{m_{1}} e^{\alpha_{2} z}+\ldots+\frac{m_{n}}{m_{1}} e^{\alpha_{n} z}$ has positive real frequencies $\alpha_{2}<\ldots<\alpha_{n}$ which are linearly independent over the rationals. Thus, from [14, Theorem 6], we have that $\sigma_{0} \in R_{S_{1}}$ if and only if (3.3) is satisfied. Now, by Remark 3.1, we have that $\sigma_{0} \in R_{S_{1}}$ if and only if $\sigma_{0} e^{-i \varphi} \in R_{S}$, and the result follows.

As a consequence of the result above, given two real numbers $\sigma_{0}<\sigma_{1}$, it is now immediate that the line segment $\left\{z \in \mathbb{C}: \sigma_{0}<z e^{i \varphi}<\sigma_{1}\right\}$ is contained in $R_{S}$ if and only if

$$
1 \leq \sum_{j=2}^{n}\left|\frac{m_{j}}{m_{1}}\right| e^{\alpha_{j} x} ; \quad\left|\frac{m_{k}}{m_{1}}\right| e^{\alpha_{k} x} \leq 1+\sum_{j=2, j \neq k}^{n}\left|\frac{m_{j}}{m_{1}}\right| e^{\alpha_{j} x}, k=2 \ldots, n
$$

for every $x \in\left(\sigma_{0}, \sigma_{1}\right)$. This characterization is analogous to that of $[7$, Theorem 1].

Hence the study of inequalities (3.4) is essential to identify the boundary points of the set $R_{S}$. In this respect, under the same conditions as the proposition above, we easily deduce from [7, Lemma 2] that if $z_{0}$ is a boundary point of $R_{S}$, then $z_{0} e^{i \varphi} \in \mathbb{R}$ satisfies all the inequalities (3.4) and only one of them is an equality. Likewise, by [7, Theorem 3], we can assure that the extreme points of the set $R_{S}$, denoted above as $a_{S}$ and $b_{S}$, are not isolated points in the set $R_{S}$.

Concerning this subject, let $\left[x_{1} e^{-i \varphi}, x_{2} e^{-i \varphi}\right]$ and $\left[x_{3} e^{-i \varphi}, x_{4} e^{-i \varphi}\right]$, with $x_{1}<x_{2}<x_{3}<x_{4}$, be two disjoint line segments contained in $R_{S}$. From now on, we will say that the interval $\left(x_{2} e^{-i \varphi}, x_{3} e^{-i \varphi}\right)$ is a gap of $R_{S}$ when no point of the form $x e^{-i \varphi}$ with $x_{2}<x<x_{3}$ belongs to the set $R_{S}$. In this sense, the fact that $R_{S}$ does not consist of one segment depends on the exact number of real solutions of any of the $n-2$ equations

$$
\left|\frac{m_{k}}{m_{1}}\right| e^{\alpha_{k} x}=1+\sum_{j=2, j \neq k}^{n}\left|\frac{m_{j}}{m_{1}}\right| e^{\alpha_{j} x}, k=2 \ldots, n-1 .
$$

In this respect, by virtue of [7, Lemma 8 and Theorem 9], notice that each equation (3.5) has at most two real solutions which are in $R_{S_{1}} \subset\left[a_{S_{1}}, b_{S_{1}}\right]$ 
and they are candidates of the boundary points of $R_{S_{1}}$. In fact, if we denote as $A_{k} \subset \mathbb{R}, 2 \leq k \leq n-1$, the set of points satisfying the $k$-th nonstrict inequality from (3.5), then there always exist $c_{k} \leq d_{k}$ such that $A_{k}=$ $\left(-\infty, c_{k}\right] \cup\left[d_{k}, \infty\right)$, and the boundary points of $R_{S_{1}}$ appear when $c_{k}<d_{k}$ in view of the fact that

$$
R_{S_{1}}=\left[a_{S_{1}}, b_{S_{1}}\right] \cap \bigcap_{k=2}^{n-1} A_{k}=\left[a_{S_{1}}, b_{1}\right] \cup\left[a_{2}, b_{2}\right] \cup \ldots \cup\left[a_{l-1}, b_{l-1}\right] \cup\left[a_{l}, b_{S_{1}}\right],
$$

where $a_{S_{1}}<b_{1}<a_{2}<\ldots<b_{l-1}<a_{l}<b_{S_{1}}$ and $\left\{b_{1}, a_{2}, b_{2}, \ldots, a_{l-1}, b_{l-1}, a_{l}\right\}$ $\subset\left\{c_{2}, d_{2}, \ldots, c_{n-1}, d_{n-1}\right\}$ (with $c_{k}<d_{k}$ ). Indeed the following theorem, which is now deduced from [7, theorems 9 and 10], gives a precise description of the set $R_{S}$ and, in fact, it establishes a bound for the number of gaps of $R_{S}$ and the reason for which these gaps are produced. Furthermore, we provide a sufficient condition which guarantees that the set $R_{S}$ consists of one segment.

Theorem 3.3. Let $S(z)=m_{1} e^{\gamma_{1} z}+m_{2} e^{\left(\gamma_{1}+\alpha_{2} e^{i \varphi}\right) z}+\ldots+m_{n} e^{\left(\gamma_{1}+\alpha_{n} e^{i \varphi}\right) z}$, $n \geq 2$, be an exponential polynomial of type (3.1) with $0<\alpha_{2}<\ldots<\alpha_{n}$ linearly independent over the rationals.

i) $R_{S}$ is either $\left[a_{S}, b_{S}\right]$ or the union of at most $n-1$ disjoint nondegenerate line segments. In the latter case, the gaps of $R_{S}$ are associated with those equations (3.5) which have two real solutions, i.e. $\left(x e^{-i \varphi}, y e^{-i \varphi}\right)$ is a gap of $R_{S}$ if and only if $x=b_{j}$ and $y=a_{j+1}$, where $1 \leq j \leq l-1$ and the $a_{j}$ 's and $b_{j}$ 's are considered above.

ii) If $\left|m_{1}\right|=\left|m_{2}\right|=\ldots=\left|m_{n}\right|$, then $R_{S}=\left[a_{S}, b_{S}\right]$, i.e. $R_{S}$ consists of one segment.

Finally, in the more general case of an exponential polynomial $P(z)$ with distinct complex frequencies (not necessarily co-linear), according to Theorem 2.1, we must consider the union of $q$ sets of type (3.2), where $q$ is the number of sides of the convex polygon $C_{P}$. In this manner, let $R_{P}$ denote the union of those sets, and let us introduce the following notation.

Notation 3.4. Consider $P(z)=S_{1}(z)+\ldots+S_{q}(z)$ of type (1.1), with $S_{r}(z)$ an exponential polynomial such that the complex conjugates of its frequencies lie on the side $l_{r}$ of the polygon $C_{P}$, where $r=1, \ldots, q$. If that is the case, let $\mathcal{C}_{I}$ denote the set of those $P(z)$ for which each $S_{r}(z)$ can be written as an exponential polynomial of type (3.1) with $0<\alpha_{2}<\ldots<\alpha_{n}$ linearly independent over the rationals. 
Now, we deduce from Theorem 3.3 that $R_{P}$ is the union of a finite amount of disjoint nondegenerate line segments for any $P(z)$ in the class $\mathcal{C}_{I}$. Also, as a clear consequence of Theorem 2.1, the following result for this particular class of exponential polynomials holds.

Corollary 3.5. Let $P(z)=S_{1}(z)+\ldots+S_{q}(z)$ be an exponential polynomial in the class $\mathcal{C}_{I}$ where $q$ is the number of sides of the convex polygon $C_{P}$. If the coefficients of any $S_{r}(z)$ have the same absolute value, then each one of the $q$ sets of $R_{P}$ consists of one segment.

It is clear that the results above can be applied to any exponential polynomial with the same set of zeros. In this sense, in the paper [12] the authors identify the families of exponential polynomials with the same set of zeros.

Example 3.6. Consider the exponential polynomial

$$
\begin{gathered}
S(z)=e^{(-1+2 i) z}+5 e^{\left(-1+2 i+\log (2) e^{\frac{3 \pi i}{4}}\right) z}+7 i e^{\left(-1+2 i+\log (11) e^{\frac{3 \pi i}{4}}\right) z}+ \\
+17 e^{\left(-1+2 i+\log (83) e^{\frac{3 \pi i}{4}}\right) z}+25 e^{\left(-1+2 i+\log (179) e^{\frac{3 \pi i}{4}}\right) z}+ \\
+11 i e^{\left(-1+2 i+\log (601) e^{\frac{3 \pi i}{4}}\right) z}+i e^{\left(-1+2 i+\log (1193) e^{\frac{3 \pi i}{4}}\right) z} .
\end{gathered}
$$

Observe that $S(z)$ is of type (3.1) where $\gamma_{1}=-1+2 i, \varphi=\frac{3 \pi}{4}, \alpha_{1}=0$, $\alpha_{2}=\log 2, \alpha_{3}=\log (11), \alpha_{4}=\log (83), \alpha_{5}=\log (179), \alpha_{6}=\log (601)$ and $\alpha_{7}=\log (1193)$. Note also that $\left\{\alpha_{2}, \alpha_{3}, \alpha_{4}, \alpha_{5}, \alpha_{6}, \alpha_{7}\right\}$ is linearly independent over the rationals. Moreover, $S(z)$ is associated (see Remark 3.1) with the exponential polynomial

$$
\begin{aligned}
S_{1}(z)=1+5 e^{\log (2) z}+7 i e^{\log (11) z} & +17 e^{\log (83) z}+ \\
& +25 e^{\log (179) z}+11 i e^{\log (601) z}+i e^{\log (1193) z}
\end{aligned}
$$

which satisfies $R_{S}=\overline{\left\{e^{-i \varphi} \operatorname{Re} z: S_{1}(z)=0\right\}}$.

The extreme points of the set $R_{S_{1}}$, denoted as $a_{S_{1}}$ and $b_{S_{1}}$, are respectively determined by the equations

$$
5 \cdot 2^{\sigma}+7 \cdot 11^{\sigma}+17 \cdot 83^{\sigma}+25 \cdot 179^{\sigma}+11 \cdot 601^{\sigma}+1193^{\sigma}=1,
$$

and

$$
1+5 \cdot 2^{\sigma}+7 \cdot 11^{\sigma}+17 \cdot 83^{\sigma}+25 \cdot 179^{\sigma}+11 \cdot 601^{\sigma}=1193^{\sigma}
$$

whose solutions are $a_{S_{1}} \approx-2.358$ and $b_{S_{1}} \approx 3.544$. The distribution of zeros of $S_{1}(z)$ with imaginary part between -200 and 200 can be observed 


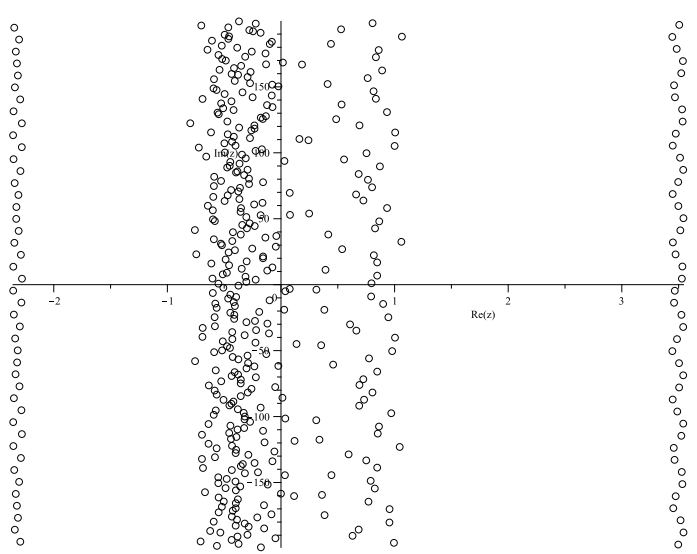

Figure 2: Distribution of zeros of the polynomial $S_{1}(z)$ defined in Example 3.6

on Figure 2. Hence the extreme points of $R_{S}$ are $a_{S} \approx-2.358 e^{-\frac{3 \pi}{4} i}$ and $b_{S} \approx 3.544 e^{-\frac{3 \pi}{4} i}$. Furthermore, equalities (3.5) are given in this case by

$$
\begin{aligned}
5 \cdot 2^{\sigma} & =1+7 \cdot 11^{\sigma}+17 \cdot 83^{\sigma}+25 \cdot 179^{\sigma}+11 \cdot 601^{\sigma}+1193^{\sigma}, \\
7 \cdot 11^{\sigma} & =1+5 \cdot 2^{\sigma}+17 \cdot 83^{\sigma}+25 \cdot 179^{\sigma}+11 \cdot 601^{\sigma}+1193^{\sigma}, \\
17 \cdot 83^{\sigma} & =1+5 \cdot 2^{\sigma}+7 \cdot 11^{\sigma}+25 \cdot 179^{\sigma}+11 \cdot 601^{\sigma}+1193^{\sigma}, \\
25 \cdot 179^{\sigma} & =1+5 \cdot 2^{\sigma}+7 \cdot 11^{\sigma}+17 \cdot 83^{\sigma}+11 \cdot 601^{\sigma}+1193^{\sigma}, \\
11 \cdot 601^{\sigma} & =1+5 \cdot 2^{\sigma}+7 \cdot 11^{\sigma}+17 \cdot 83^{\sigma}+25 \cdot 179^{\sigma}+1193^{\sigma} .
\end{aligned}
$$

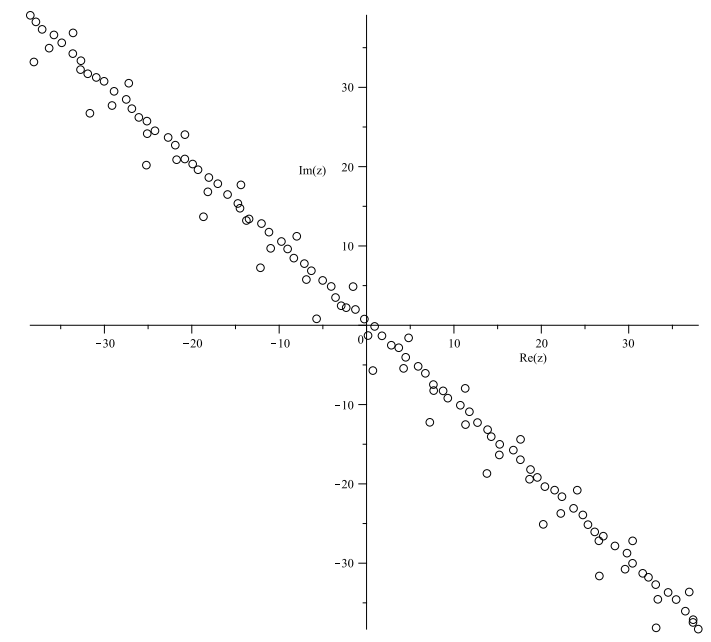

Figure 3: Distribution of zeros of the polynomial $S(z)$ defined in Example 3.6

We next use Theorem 3.3 in order to calculate the disjoint line segments of $R_{S}$. The equality in $(3.6)$ is reached at $d_{1} \approx-2.278$ and $d_{2} \approx-0.824$. The equality in $(3.10)$ is reached at $d_{3} \approx 1.078$ and $d_{4} \approx 3.443$. Finally, 
equalities (3.7), (3.8) and (3.9) have no real solutions. Therefore, the gaps in $R_{S_{1}}$ are $\left(d_{1}, d_{2}\right)$ and $\left(d_{3}, d_{4}\right)$, as we observe in Figure 2. Consequently, the gaps in $R_{S}$ are determined by the line segments $\left(d_{1} e^{-\frac{3 \pi}{4} i}, d_{2} e^{-\frac{3 \pi}{4} i}\right)$ and $\left(d_{3} e^{-\frac{3 \pi}{4} i}, d_{4} e^{-\frac{3 \pi}{4} i}\right)$. Figure 3 presents the distribution of zeros of $S(z)$ with the imaginary and real part between -40 and 40 .

\section{Acknowledgements}

The second author's research was partially supported by MICIU of Spain under project number PGC2018-097960-B-C22, and by Generalitat Valenciana under project GV/2015/035. The authors thank Professor Z. Szafraniec for his valuable comments on an earlier version of our manuscript.

\section{References}

[1] C.E. Avellar, J.K. Hale, On the zeros of exponential polynomials, J. Math. Anal. Appl., 73, (1980) 434-452.

[2] E. Dubon, G. Mora, J.M. Sepulcre, J.I. Ubeda, T. Vidal, On the real projection of the zeros of $1+2^{s}+\ldots+n^{s}$, Rev. R. Acad. Cienc. Exactas Fís. Nat. Ser. A Math. RACSAM, 108, (2014) 317-333.

[3] E. Dubon, J.M. Sepulcre, On the zeros of Dirichlet polynomials and their applications to the non-lattice fractal strings, Exp. Math., 23 (1), (2014) 13-24.

[4] E. Dubon, J.M. Sepulcre, On the existence of equivalent Dirichlet polynomials whose zeros preserve a topological property, Int. J. Number Theory, 14 (3), (2018) 713-725.

[5] H.M. Farag, Dirichlet truncations of the Riemann zeta-function in the critical strip possess zeros near every vertical line, Int. J. Number Theory, 4 (4), (2008) 653-662.

[6] R.E. Langer, On the zeros of exponential sums and integrals, Bull. Amer. Math. Soc., 37, (1931) 213-239.

[7] G. Mora, J.M. Sepulcre, T. Vidal, On the existence of exponential polynomials with prefixed gaps, Bull. Lond. Math. Soc., 45 (6), (2013) 1148-1162. 
[8] C.J. Moreno, The zeros of exponential polynomials (I), Compos. Math., 26 (1), (1973) 69-78.

[9] G. Pólya, Geometrisches ber die Verteilung der Nullstellen gewisser ganzer transzendenter Funktionen; Munch. Sitzungsber., 50, (1920) 285-290.

[10] J.F. Ritt, Algebraic combinations of exponentials, Trans. Amer. Math. Soc., 31, (1929) 654-679.

[11] J.M. Sepulcre, T. Vidal, A new approach to obtain points of the closure of the real parts of the zeros of the partial sums $1+2^{z}+\ldots+n^{z}, n \geq 2$, Kybernetes, 41, (2012) 96-107.

[12] J.M. Sepulcre, T. Vidal, Equivalence classes of exponential polynomials with the same set of zeros, Complex Var. Elliptic Equ., 61 (2), (2016) 225-238.

[13] J.M. Sepulcre, T. Vidal, On the non-isolation of the real projections of the zeros of exponential polynomials, J. Math. Anal. Appl., 437 (1), (2016) 513-525.

[14] J.M. Sepulcre, On the result of invariance of the closure set of the real projections of the zeros of an important class of exponential polynomials, Journal of Function Spaces, vol. 2016, Article ID 3605690.

[15] J.D. Tamarkin, The zeros of certain integral functions, J. Lond. Math. Soc., 2, (1927) 66-69.

[16] J.D. Tamarkin, Some general problems of the theory of ordinary linear differential equations and expansions of an arbitrary function in series of fundamental functions, Math. Z., 27, (1928) 1-54.

[17] C.E. Wilder, Expansion problems of ordinary linear differential equations with auxiliary conditions at more than two points, Trans. Amer. Math. Soc., 18, (1917) 415-442. 